\title{
SOME VECTOR BUNDLES ON JACOBIAN VARIETIES ${ }^{1}$
}

\author{
GEORGE R. KEMPF
}

\begin{abstract}
Picard bundles on a Jacobian variety are invariant under some subgroups of its fundamental group.
\end{abstract}

Interesting examples of vector bundles on abelian varieties occur in the study of the inversion of abelian integrals on a Riemann surface. The purpose of this note is to examine one property of these bundles.

Let $X=V / L$ be a complex torus where $L$ is a lattice of maximal rank contained in a complex vector space $V$. Given a complex analytic vector bundle $W$ on $X$, one can try to find a sublattice $M \subset L$ such that $(\lambda) \pi^{*} W$ is an analytically trivial bundle where $\pi^{*} W$ is the pull-back of $W$ via the quotient map $\pi: V / M \rightarrow V / L=X$. Let us say $W$ is invariant under $M$ if condition $(\lambda)$ is satisfied.

In particular, any bundle is invariant under the trivial lattice $\{0\}$, as any complex analytic bundle on $V$ is trivial [1]. If $W$ is a line bundle on $X$ and $W$ is invariant under $M$, then $M$ is an isotropic sublattice of $L$ with respect to the Riemann form of $W$ (see [4], for instance). Conversely, if $M$ is an isotropic sublattice of $L$ and the homomorphism $M \otimes \mathbf{C} \rightarrow V$ is injective, the line bundle $W$ is invariant under $M$. Furthermore, the second condition on $M$ is always satisfied if $W$ is a nondegenerate line bundle. In this case, if $M$ is a maximal isotropic sublattice, one knows that $M \otimes \mathrm{C} \stackrel{\approx}{\longrightarrow}$ and $V / M$ is isomorphic to a product of $\mathbf{C}^{*}$ 's $\left(\mathbf{C}^{*}=\mathbf{C}-\{0\}\right)$.

Proposition. Let $\Theta$ be the cohomology class of a divisor on an abelian variety $X=V / L$. Assume $W$ is an analytic vector bundle on $X$ such that the ith Chern class of $W$ equals $\Theta^{i} / i$ ! in the cohomology ring of $X$. If

(1) $M$ is an isotropic sublattice for the Riemann form of $\Theta$,

(2) the homomorphism $M \otimes \mathbf{C} \rightarrow V$ is injective, and

(3) twice the rank of $M$ is less than or equal to the rank of $W$, then $W$ is invariant under $M$.

Proof. $V / M$ is a Stein manifold because it is a product of $\mathbf{C}^{*}$ 's with $\mathbf{C}^{\prime}$ 's by (2). Thus, by Grauert's theorem, we need only check that $\pi^{*} W$ is topologically trivial. The $i$ th Chern class of $\pi^{*} W$ equals $\left[\pi^{*} \Theta\right]^{i} / i$ ! by assumption. By (1), $\pi^{*} \Theta$ is the zero cohomology class. Let $Y$ be the maximal compact subgroup

Received by the editors December 3, 1974.

AMS (MOS) subject classifications (1970). Primary 32L05, 14K30.

Key words and phrases. Vector bundle, Jacobian variety.

${ }^{1}$ Partly supported by NSF Grant GP 36269 and Sloan Foundation Fellowship. 
of $V / M$. Then $Y$ is a product of rank of $M$ number of circles. $Y$ is homotopic to $V / M$. Thus, to show that $\pi^{*} W$ is topologically trivial, we need only note that its restriction to $Y$ is topologically trivial. This fact follows because the restriction to $Y$ has zero Chern classes and large enough rank [5]. Q.E.D.

I will now apply the above Proposition to the intersecting bundles on Jacobians. These bundles have been studied by Mattuck [2], [3] and Schwarzenberger [6]. Let $S$ be a compact Riemann surface with fixed point $P$. Let $J$ be the Jacobian variety of $S$. For any interger $d$, let $\mathcal{L}_{d}$ be the analytic bundle on $S \times J$ with $\mathcal{E}_{d} \mid S \times\{0\}$ isomorphic to $\Theta(d P)$ and $\left.\mathcal{E}_{d}\right|_{\{P\} \times J} \approx \Theta_{J}$ such that $\left.\mathcal{L}\right|_{S \times\{j\}}$ runs through all line bundles on $S$ of degree $d$ in a universal manner as $j$ varies in $J$. Let $g$ denote the genus of $S$. If $d \geq 2 g-1$, the direct image

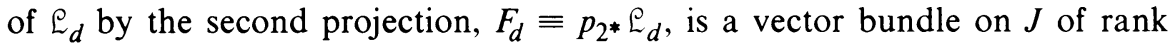
$d-g+1$.

These bundles $F_{d}$ are the bundles of interest in this paper. Mattuck has determined that the $i$ th Chern class of any $F_{d}$ is the cohomology class of the subvariety $W_{i}$ of $J$. Here, $W_{i}$ denotes the set-theoretic image of $g-i$ copies of $S$ under the sum of Jacobian mappings applied to each projection. Let $\Theta$ be the cohomology class of the ample divisor $W_{1}$. By Poincarés intersection relations, the cohomology class of $W_{i}$ is $\Theta^{i} / i$ ! . Combining the above results with the Proposition, we may conclude:

THEOREM. $A$ bundle $F_{d}$ is invariant under any sublattice of the fundamental group of $J$ which is isotropic for the Riemann form of $\Theta$.

Having this abstract theorem for the existence of a trivialization of a bundle, one may ask several questions: Is there any reasonable explicit way to trivialize the bundle? What kinds of factors of automorphy determine the bundle on the abelian variety, especially now that we can choose some to be the identity?

\section{REFERENCES}

1. H. Grauert, Analytische Faserungen über holomorph-vollständigen Räumen, Math. Ann. 135 (1958), 263-273. MR 20 \#4661.

2. A. Mattuck, Symmetric products and Jacobians, Amer. J. Math. 83 (1961), 189-206. MR 26 \# 122 .

3. - Secant bundles on symmetric products, Amer. J. Math. 87 (1965), 779-797. MR 33 \#7345.

4. D. Mumford, Abelian varieties, Tata Inst. Fund. Res. Studies in Math., no. 5, Oxford Univ. Press, London, 1970. MR 44 \#219.

5. F. P. Peterson, Some remarks on Chern classes, Ann. of Math. (2) 69 (1959), 414-420. MR 21 \# 1593.

6. R. L. E. Schwarzenberger, Jacobians and symmetric products, Illinois J. Math. 7 (1963), 257-268. MR 27 \# 1444.

Department of Mathematics, The Johns Hopkins University, Baltimore, Maryland 21218 